Revue des patrimoines

\title{
Le "module médiéval" de l'abbaye classique Sainte-Marie ou Notre-Dame de la Sagne à Vielmur-sur-Agout (Tarn)
}

\section{Dominique Watin-Grandchamp}

\section{(2) OpenEdition \\ Journals}

Édition électronique

URL : http://journals.openedition.org/insitu/1208

DOI : $10.4000 /$ insitu. 1208

ISSN : 1630-7305

Éditeur

Ministère de la culture

Référence électronique

Dominique Watin-Grandchamp, « Le "module médiéval" de l'abbaye classique Sainte-Marie ou NotreDame de la Sagne à Vielmur-sur-Agout (Tarn) », In Situ [En ligne], 2 | 2002, mis en ligne le 25 janvier 2012, consulté le 02 mai 2019. URL : http://journals.openedition.org/insitu/1208 ; DOI : 10.4000/ insitu. 1208

Ce document a été généré automatiquement le 2 mai 2019.

\section{(c) (i) (3)}

In Situ Revues des patrimoines est mis à disposition selon les termes de la licence Creative Commons Attribution - Pas d'Utilisation Commerciale - Pas de Modification 4.0 International. 


\title{
Le "module médiéval" de l'abbaye classique Sainte-Marie ou Notre- Dame de la Sagne à Vielmur-sur- Agout (Tarn)
}

\author{
Dominique Watin-Grandchamp
}

1 Une première demande de protection au titre des Monuments historiques concernant une partie du corps de logis sur la cour d'entrée et l'église abbatiale, aujourd'hui paroissiale, de abbaye de Notre-Dame de la Sagne a été examinée par la COREPHAE en 1988. Devant la complexité du dossier, parcelles appartenant à des particuliers, à la commune (église) et au département (collège) et l'aspect "bétonné" de certaines élévations, cette commission a demandé, avant de se déterminer sur une protection de l'édifice, un complément d'information sur l'ensemble du couvent. Des investigations menées dans le bâtiment contigu au mur ouest de l'église ont permis la découverte d'éléments médiévaux. L'ensemble de l'édifice a été inscrit sur l'inventaire supplémentaire des Monuments historiques le 26 décembre 1995, puis, grâce à ces découvertes, les bâtiments abbatiaux, l'église Saint-Germinien, les jardins, dépendances et édicules compris dans l'ancien enclos abbatial ont été classés en 1996.

\section{Survol historique de l'abbaye}

2 L'implantation d'une abbaye bénédictine à Vieilmur-sur-Agout, située au bord de la rivière Agout, à $10 \mathrm{~km}$ de Lautrec, remonterait au $\mathrm{X}^{\mathrm{e}}$ siècle. Une paroisse Saint-Clément sise au lieu-dit "Veteris muris" est citée dans une charte de donation de Raymond Pons comte de Toulouse en 936. Cependant, ce sont des membres de la famille Lautrec qui sont considérés comme ses fondateurs. En 1038 Isarn de Lautrec et son frère Frotard, évêque d'Albi, soumettent le couvent à Notre-Dame du Puy et Guisle, première abbesse de Vielmur, est la fille d'Isarn. Jusqu'à la fin du XIVe siècle, l'abbaye reste sous la protection de cette illustre famille et de ses collatéraux : Cardaillac, Lévis et Alaman, d'où sont issues 
la plupart de ses abbesses. Au XVe siècle la puissance des Lautrec diminue et l'abbatiat n'est plus l'apanage de leurs seules filles.

La guerre de Cent Ans, puis les guerres de Religion n'épargnent pas l'abbaye dont on ne connaît pas les dispositions d'origine. Elle est incendiée par les protestants en 1568 et les moniales sont dispersées. Elles réintègrent les lieux en 1576 sous l'abbatiat de Madeleine d'Arpajon mais l'abbaye se relève difficilement de ses ruines et, jusqu'en 1665, 11 moniales y vivent dans des conditions difficiles. C'est à l'abbesse Jeanne Louise d'Arpajon (1665-1700), nièce du maréchal de Thémines, que l'on doit le début d'une grande de campagne de reconstruction des bâtiments abbatiaux qui se poursuit jusqu'en 1780. En 1667-1690 on élève les vastes ailes nord et sud qui se développent autour d'un nouveau cloître et, de 1778 à 1780, l'aile ouest. A la même époque l'église, agrandie à la fin du XVI ${ }^{\mathrm{e}}$ siècle, reçoit un nouveau décor. Vendue comme bien national en 1793, la propriété est morcelée au début du XIX ${ }^{e}$ siècle. L'installation d'un collège dans la partie sud en 1954 impose la restructuration des espaces intérieurs.

\section{Le "module médiéval"}

Figure 1

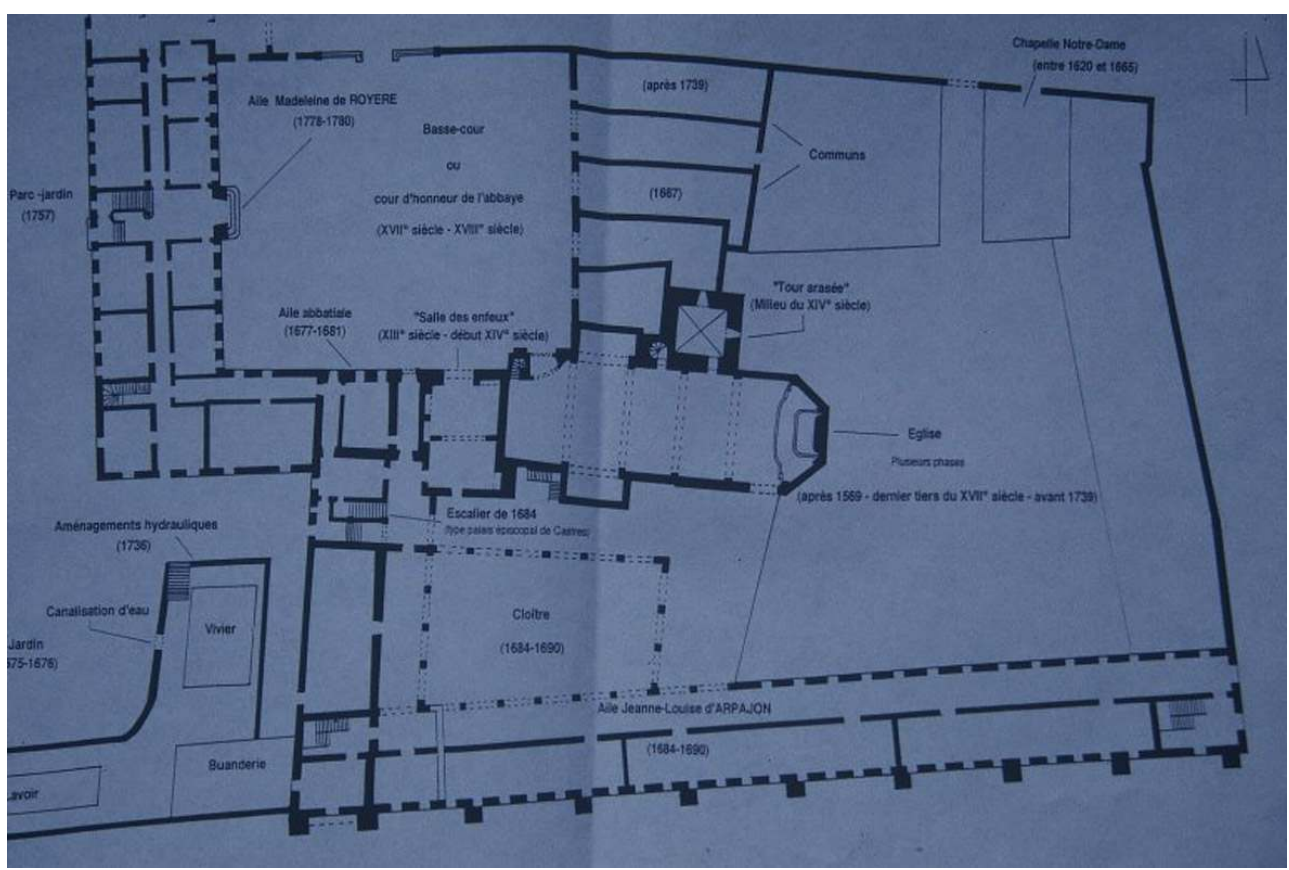

Proposition de restitution d'ensemble de l'ancienne abbaye Notre-Dame de la Sagne à Vielmur (Tarn), par J. Dauzat

Phot. D. Watin-Grandchamp @ C CRMH Midi-Pyrénées, 1995

Les constructions de l'abbaye classique se développent en forme de "Z" du sud au nord autour de l'église. La cour d'honneur au nord est bordée, à l'est, par des communs des XVII et XVIII ${ }^{e}$ siècles, à l'ouest, par l'aile élevée par Madeleine de Royère de 1778 à 1780 et au sud par les constructions de 1677-1681 qui se prolongent en retour au sud pour rejoindre les bâtiments de 1684-1690 en partie détruits. Cet ensemble détermine l'espace occupé par le cloître (fig. $\mathbf{n}^{\circ} \mathbf{1}$ ). 
5 L'aile sud de la cour nord, face au bourg, a donc bien été élevée dans la seconde moitié du XVII ${ }^{e}$ siècle. Cependant, on remarque que les deux travées, comprises entre le portail de l'église et celui des bâtiments abbatiaux, sont plus élevées que l'ensemble de l'aile et que les deux niveaux de fenêtres ne sont pas au même niveau que celles qui suivent.

\section{Figure 2}

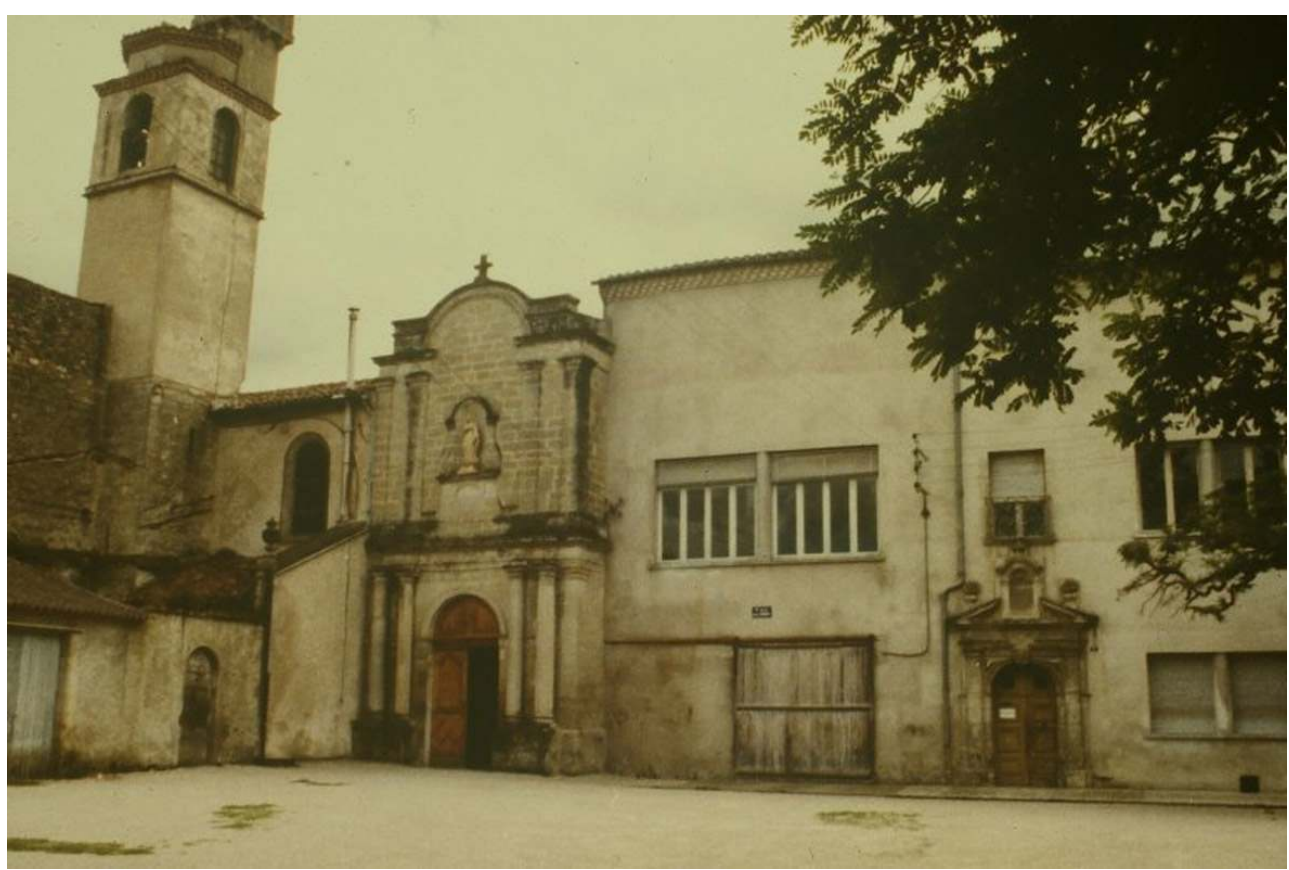

Vue d'ensemble sur cour de l'actuel collège et de l'église Notre-Dame

Phot. D. Watin-Grandchamp @ CRMH Midi-Pyrénées, 1995

6 (fig. $\mathbf{n}^{\circ}$ 2) Dans les années 1950 la façade de cet ensemble a été fortement modifiée par l'agrandissement de la porte d'entrée, l'ouverture d'une baie moderne remplaçant les fenêtres du premier niveau et la suppression de celles du second niveau. Un crépis en ciment a achevé sa dénaturation. 
Figure 3

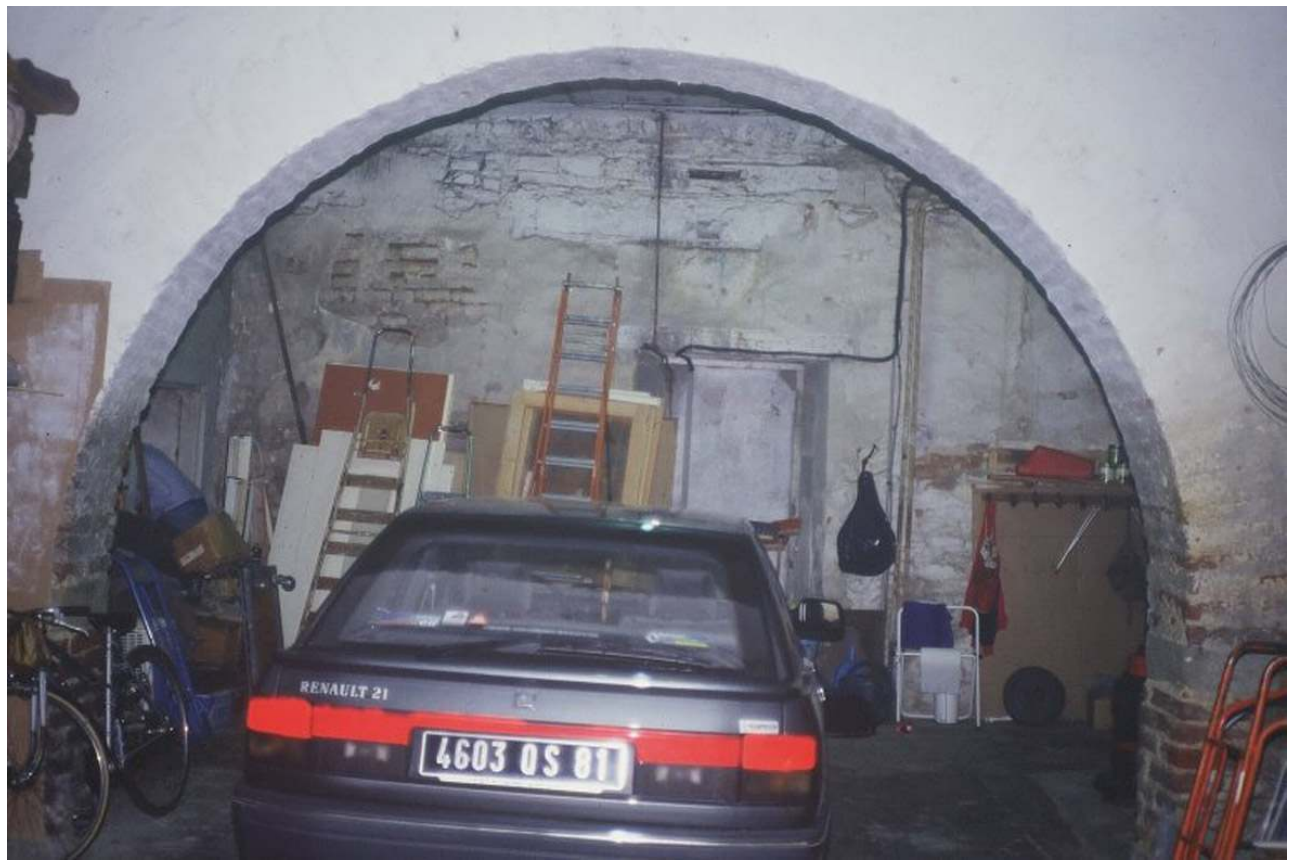

Vue d'ensemble de la salle dite des enfeus, actuel garage-débarras accolé à l'Ouest de l'église Phot. D. Watin-Grandchamp (c) CRMH Midi-Pyrénées, 1995

7 (fig. $\mathbf{n}^{\circ}$ 3) Cet espace composé de trois niveaux, dont le premier est désigné comme "ancien parloir" dans les procès-verbaux de visite de l'époque révolutionnaire, aujourd'hui garage-débarras a attiré l'attention du service des Monuments historiques, d'autant que la porte d'entrée conserve l'amorce d'un arc en plein cintre tronqué et deux impostes décorées d'une frise végétale ornée d'animaux fantastiques de la première moitié du XIV siècle. 


\section{Figure 4}

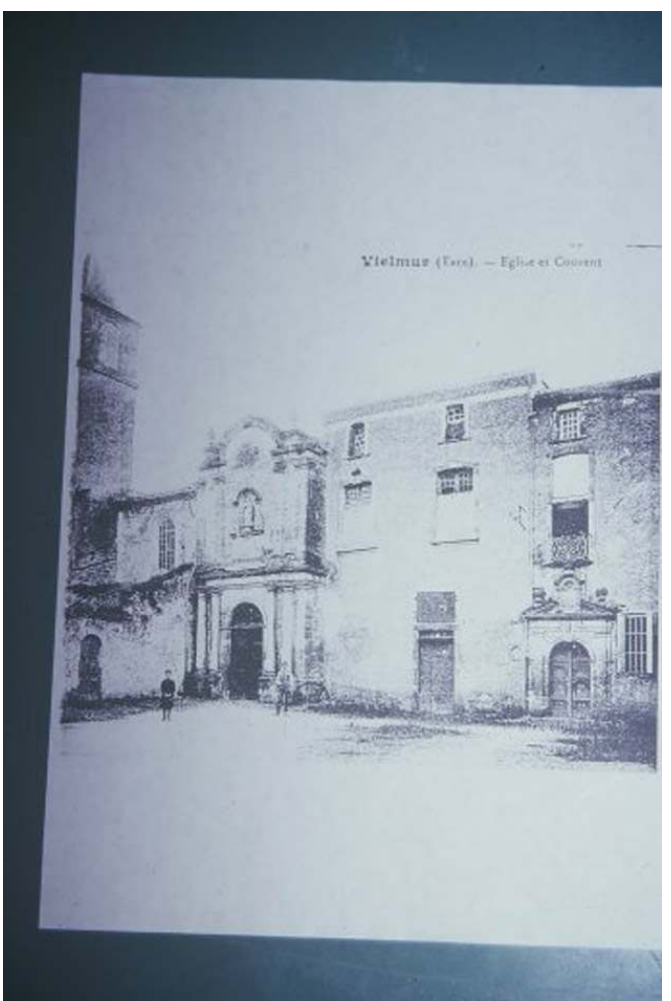

Carte postale début 20e. Elévation extérieure Nord de l'ancienne abbaye et de l'église Phot. D. Watin-Grandchamp (c) CRMH Midi-Pyrénées, 1995

\section{Figure 5}

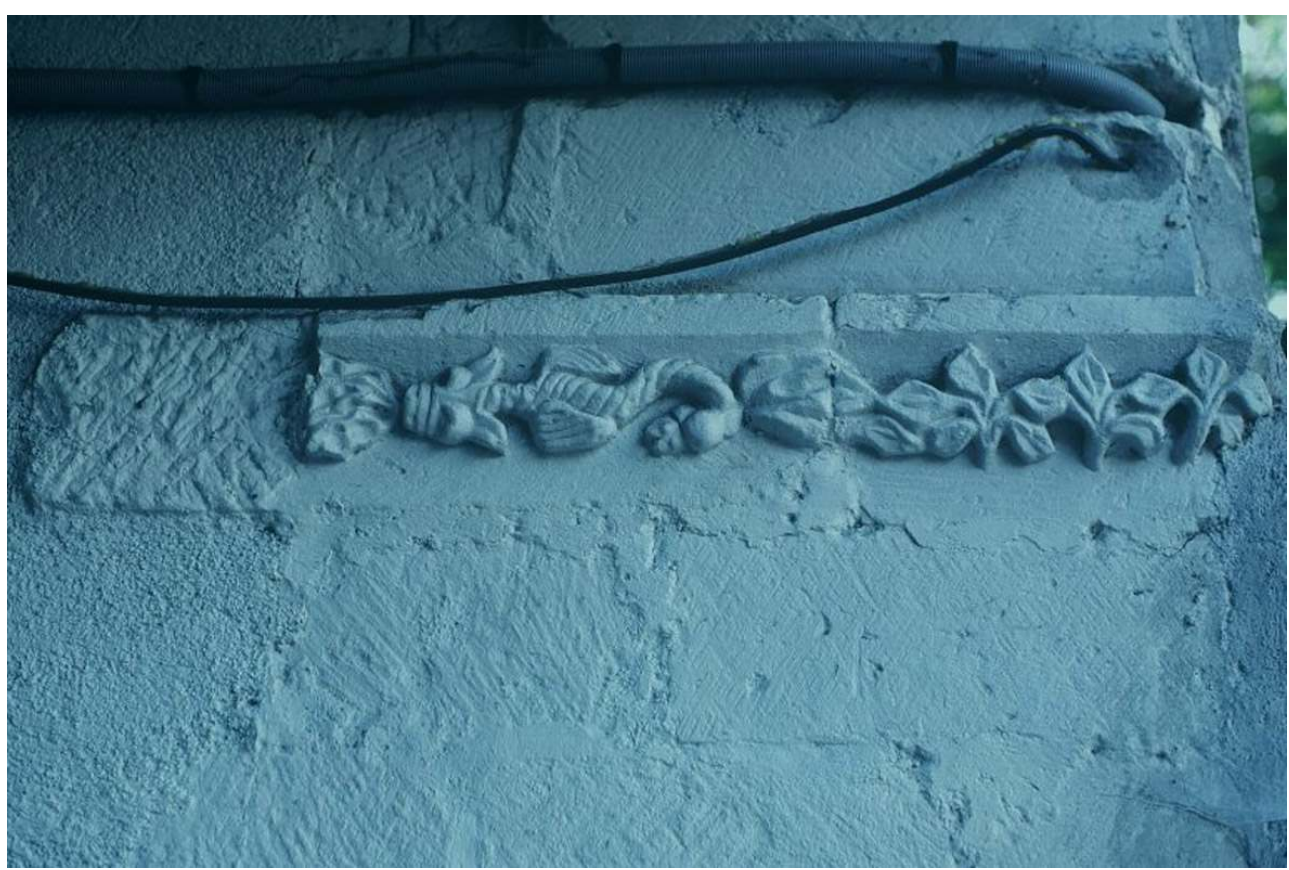

Détail dragon sculpté en imposte de l'accès à la salle dite des enfeus Phot. D. Watin-Grandchamp (c) CRMH Midi-Pyrénées, 1995 
8 Par ailleurs on savait qu'en 1858 , lors de travaux consécutifs à un procès de délimitation de parcelles, un ancien passage menant à l'église avait été mis au jour et que l'on y avait découvert, dans l'épaisseur des murs, deux "sépultures" dont l'une portait l'épitaphe de l'abbesse Poncia, inhumée en 1238. Le sarcophage et le mobilier ont aujourd'hui disparu.

9 Grâce à ces vestiges, le service des Monuments historiques obtint du collège, qui occupe ce bâtiment, le dégagement partiel du local du premier niveau, qui se révéla être une salle funéraire. Une visite approfondie des lieux permis aussi la découverte de peintures murales décoratives dans les combles.

\section{Salle des enfeux}

10 Celle-ci mesure 4,50 $\mathrm{m}$ de long sur 6,50 $\mathrm{m}$ de large. L'épaisseur des murs varie de plus d'un mètre au rez-de-chaussée à $60 \mathrm{~cm}$ pour la partie sous combles. Ses murs ouest, nord et est sont appareillés en pierre de taille de calcaire blanc à grain fin que l'on peut dater des XIII e-XIVe siècles; au sud on trouve des briques de 8 à $10 \mathrm{~cm}$ d'épaisseur datant de la même époque.

Figure 6

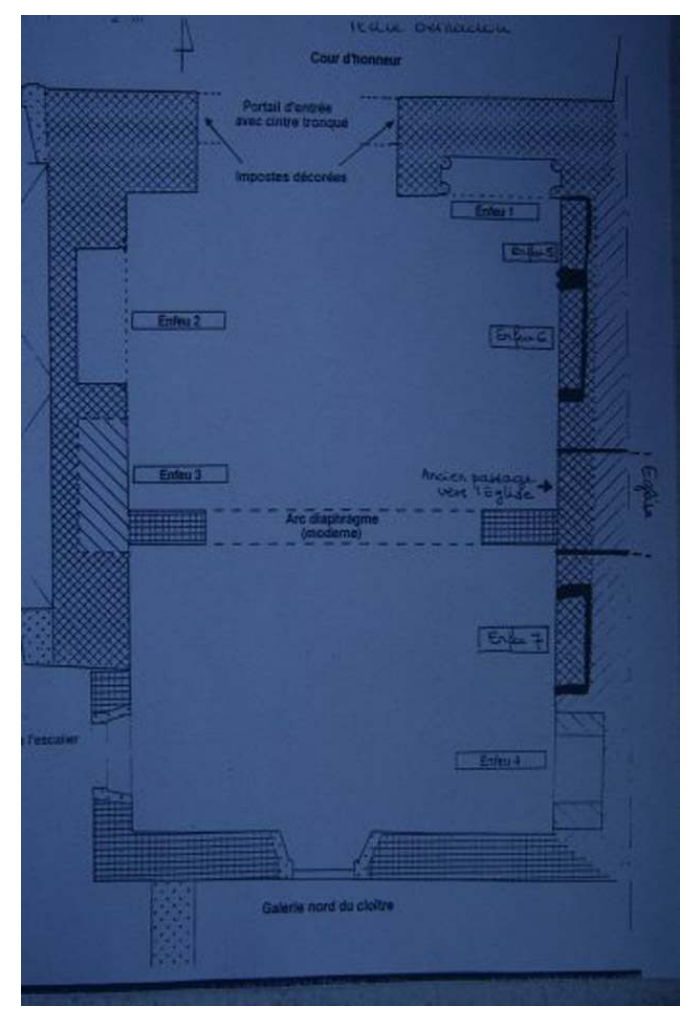

Plan sommaire de la salle dite des enfeus par J. Dauzat

Phot. D. Watin-Grandchamp @ CRMH Midi-Pyrénées, 1995

11 Les murs sont percés de sept niches (fig. $\mathbf{n}^{\circ}$ 6), certaines bouchées ou transformées en placards. Leurs dispositions, arcs en plein cintre, restes de colonnettes engagées à chapiteaux à crossettes ou à feuillages et banquettes conservées permettent de penser qu'il s'agit d'enfeux. 
Figure 7

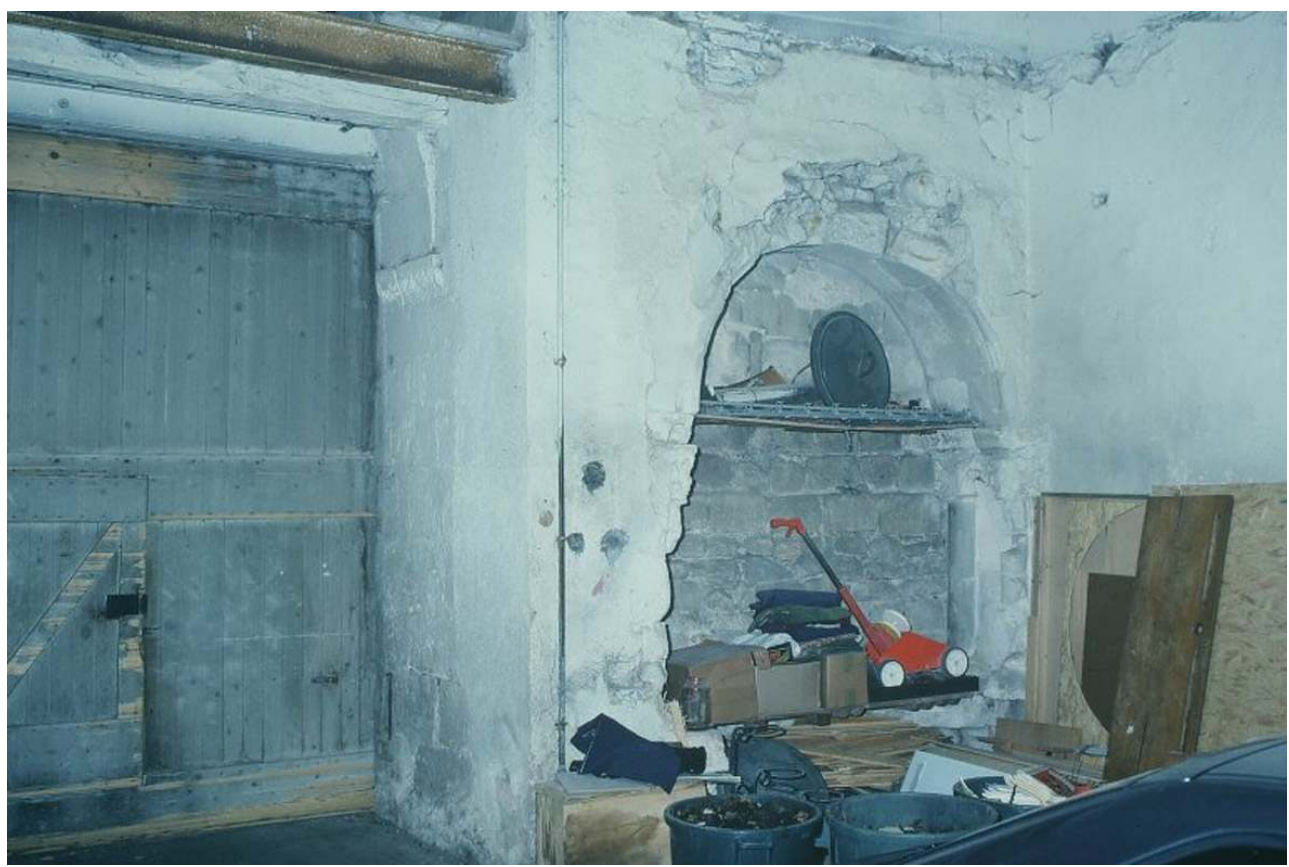

Enfeu 1, élévation Nord

Phot. D. Watin-Grandchamp @ C CRMH Midi-Pyrénées, 1995

Le $\mathrm{n}^{\circ} 1$ se trouve au revers de la façade nord, quatre sont creusés dans le mur est ( $\mathrm{n}^{\circ} 4$ à 7)) et deux dans le mur ouest ( $n^{\circ} 2$ et 3 ). L'enfeu $n^{\circ} 1$, très dégradé, est surmonté d'un arc en plein cintre orné d'un simple tore qui retombe sur deux colonnettes engagées à chapiteau à crochets caractéristiques de la seconde moitié du XIII ${ }^{e}$ siècle (fig. $\mathbf{n}^{\circ}$ 7). Les enfeux 2 et 3, mieux conservés, sont contemporains. Un double tore souligne les arcatures soigneusement appareillées et retombe sur deux colonnettes engagées.

L'enfeu 3 est en partie occulté par la retombée d'un arc diaphragme moderne supportant le plafond actuel, le 2 conserve des traces de peintures murales. 
Figure 8

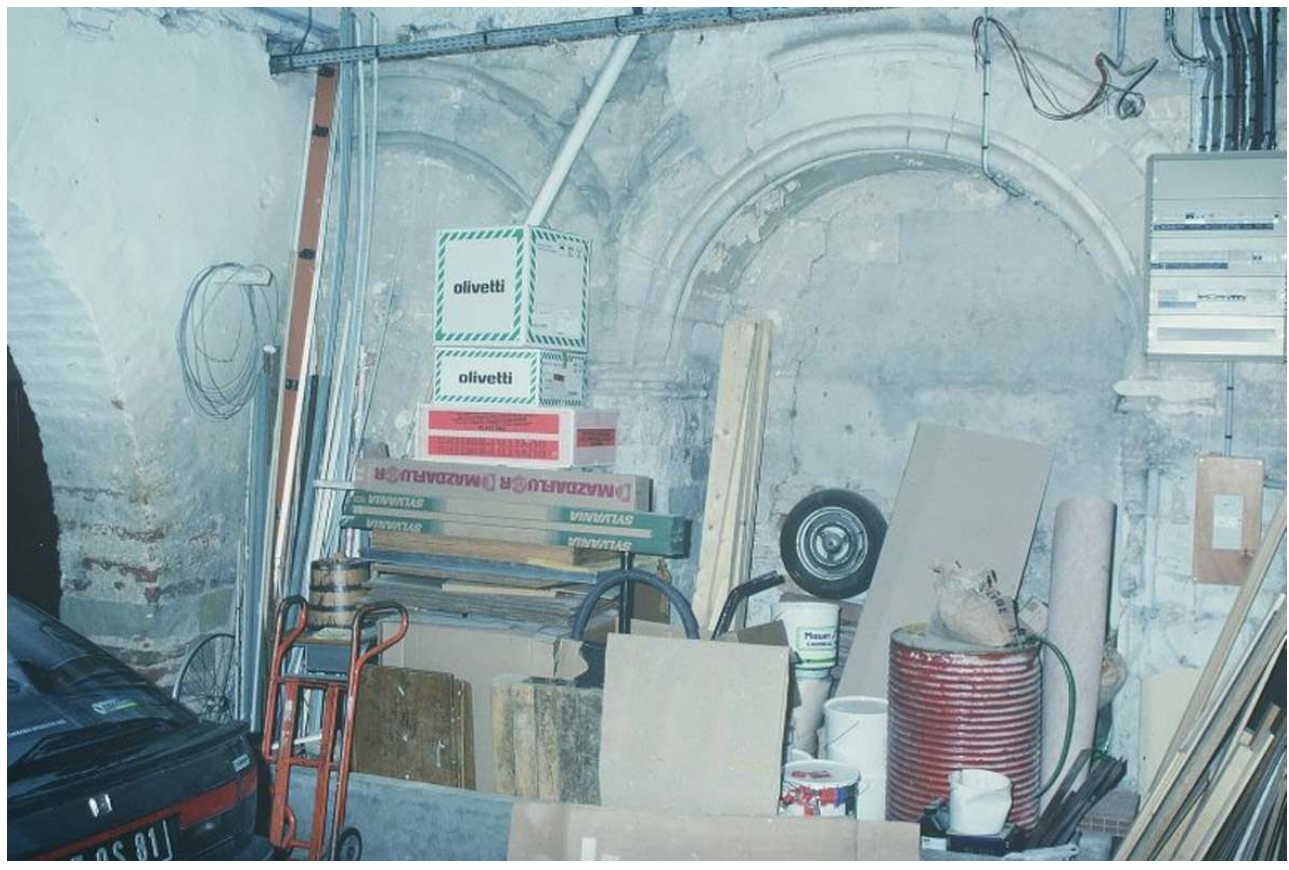

Enfeus 2 et 3, élévation Ouest

Phot. D. Watin-Grandchamp (C) CRMH Midi-Pyrénées, 1995

\section{Figure 9}

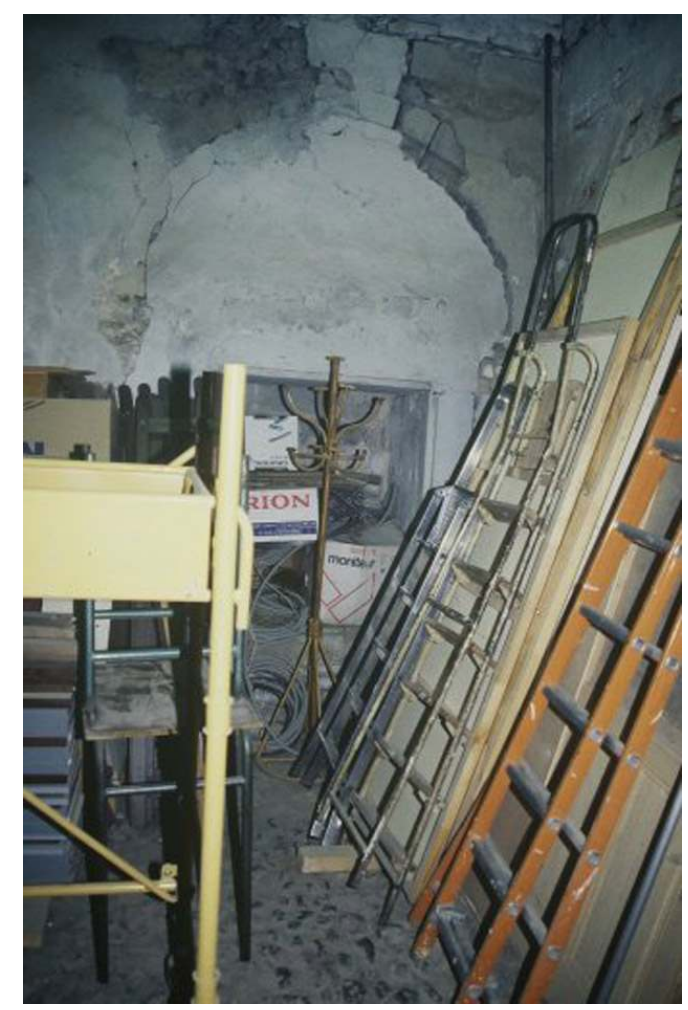

Enfeu 4 transformé en placard, élévation Est

Phot. D. Watin-Grandchamp ( C CRMH Midi-Pyrénées, 1995 

placard : cette diposition a protégé une partie du décor peint qui onsé placard : cette disposition a protégé une partie du décor peint qui orne l'intrados de l'arc.

Figure 10

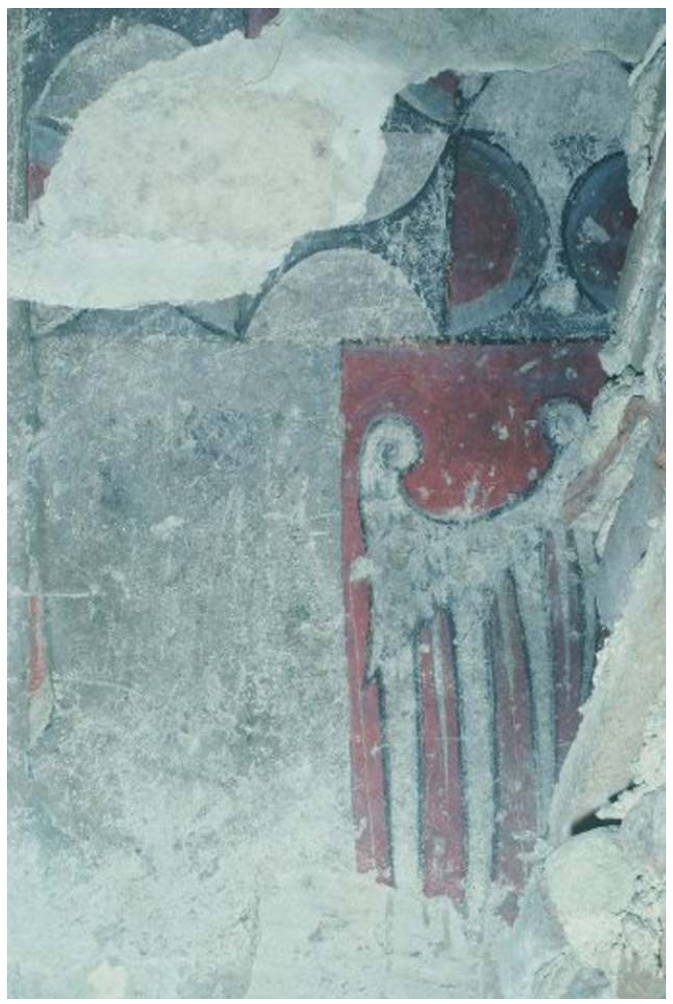

Enfeu 4 détail : blason timbrant l'intrados de l'arc : demi vol de la famille d'Alaman Phot. D. Watin-Grandchamp (c) CRMH Midi-Pyrénées, 1995

On y voit des motifs géométriques noirs et rouges traités en bandes et le blason de la famille des Alaman alliée à celle des Lautrec (fig. $\mathbf{n}^{\circ}$ 10). Au fond de la niche subsistent des vestiges ténus d'une draperie soutenue par deux anges. La présence de trois autres enfeux a été repérée dans le mur est.

Les enfeux 1,4,5,6,7 ainsi que les deux sépultures découvertes en 1858 sont situés à proximité de l'élévation ouest de l'église. Ils pourraient faire partie d'une même campagne d'aménagement à la fin du XIII ${ }^{e}$ siècle et au début du XIV ${ }^{e}$ siècle, époque de construction ou reconstructions importantes dans la région. La sculpture des enfeux 2 et 3 conduit à une datation plus tardive de quelques décennies.

On sait par les obits et les testaments conservés qu'ont été enterrés dans l'abbaye au XIV ${ }^{\mathrm{e}}$ siècle, Sicard de Lautrec en 1300, Yolande vicomtesse de Lautrec en 1318, Marquèse dame de Dourgne en 1335, Sicard de Lautrec évêque d'Agde, qui précise dans son testamen, en 1365, qu'il souhaite être enterré auprès de sa mère Marguerite de Périgueux. A cette liste on peut ajouter un membre de la famille Alaman dont on a trouvé les armes dans l'un des enfeux.

Cette salle funéraire, véritable panthéon de la famille Lautrec, est exceptionnelle en MidiPyrénées, mais seuls un dégagement et une étude archéologique fine permettraient de déterminer avec précision la datation des enfeux. Cependant, lors des débats de la 
Commission supérieure, Anne Prache a fait observer que leur sculpture s'apparentait à l'œuvre manuscrite de Limoges.

\section{L'étage et les vestiges d'une salle haute dans les combles}

Figure 11

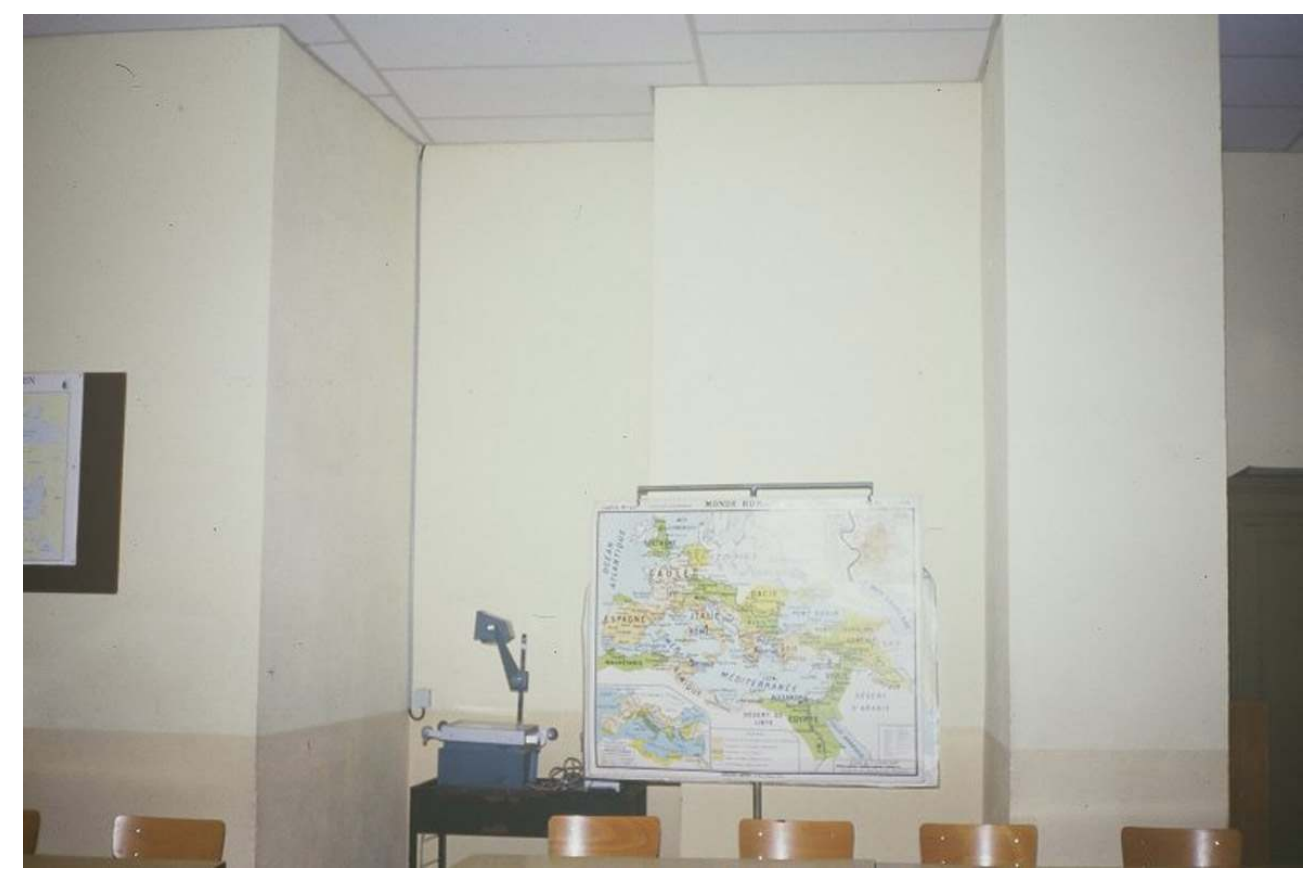

Niveau d'étage au-dessus de la salle des enfeus : salle de classe

Phot. D. Watin-Grandchamp (C) CRMH Midi-Pyrénées, 1995

19 L'étage actuel abrite une salle de classe (fig. $\left.\mathbf{n}^{\circ} \mathbf{1 1}\right)$ qui, si elle a modifié les dispositions d'origine a conservé, à l'ouest, les traces d'une cheminée monumentale et à l'est l'ébrasement d'un passage ménagé vers la tribune de l'église, ancien choeur haut des religieuses du XVIIIe siècle.

Il n'y avait plus d'accès aux combles, mais la gestion technique de la couverture du collège a nécessité l'aménagement d'un passage dans le mur ouest traversant le conduit de la cheminée. Ce passage (fig. $\mathbf{n}^{\circ} \mathbf{1 2}$ ) a permis la découverte, dans des conditions "acrobatiques", de peintures murales et de poutraisons médiévales, en particulier la trace d'un solin prouvant que le bâtiment médiéval se poursuivait vers l'ouest. Il s'agit sans doute des vestiges du décor d'une "salle haute". 
Figure 12

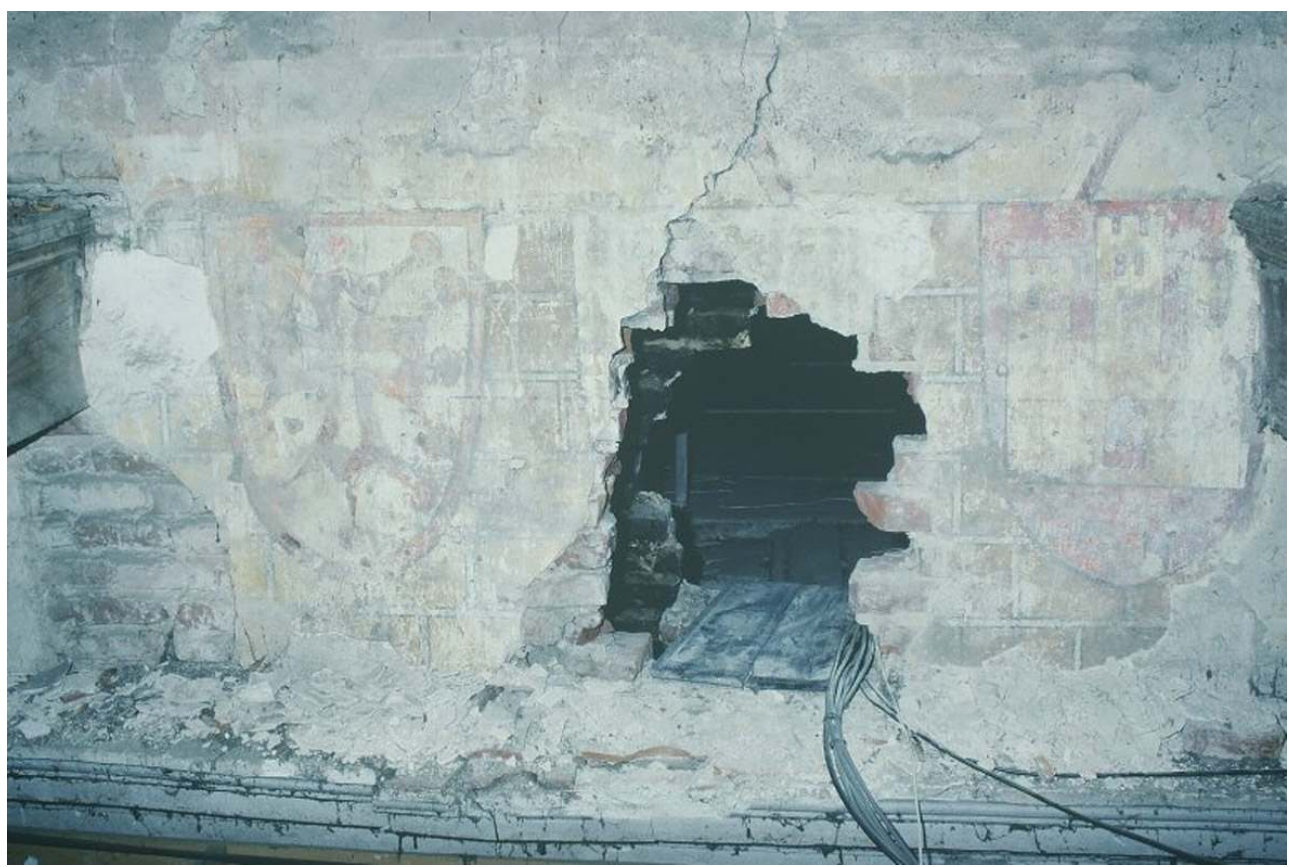

Niveau de comble actuel, frise de blasons peints

Phot. D. Watin-Grandchamp (c) CRMH Midi-Pyrénées, 1995

21 Une frise d'écus armoriés, 17 sont actuellement lisibles, se détache sur les murs l'ouest, nord et sud sur un appareil de fausse pierre jaune avec des joints blancs soulignés de noir. Le mur est a été doublé en 1844 pour séparer l'église de l'ancien parloir. La série la mieux conservée, 11 blasons, se trouve à l'ouest. Au nord, un seul est lisible, les autres ont été partiellement détruits au XVII ${ }^{e}$ siècle lors du percement des deux grandes baies. Au sud 5 sont encore lisibles. Sur ce mur on remarque une baie en plein cintre bouchée et peutêtre la trace d'une canalisation de récupération des eaux pluviales (fig. $\mathbf{n}^{\circ} \mathbf{1 3}$ ). 
Figure 13

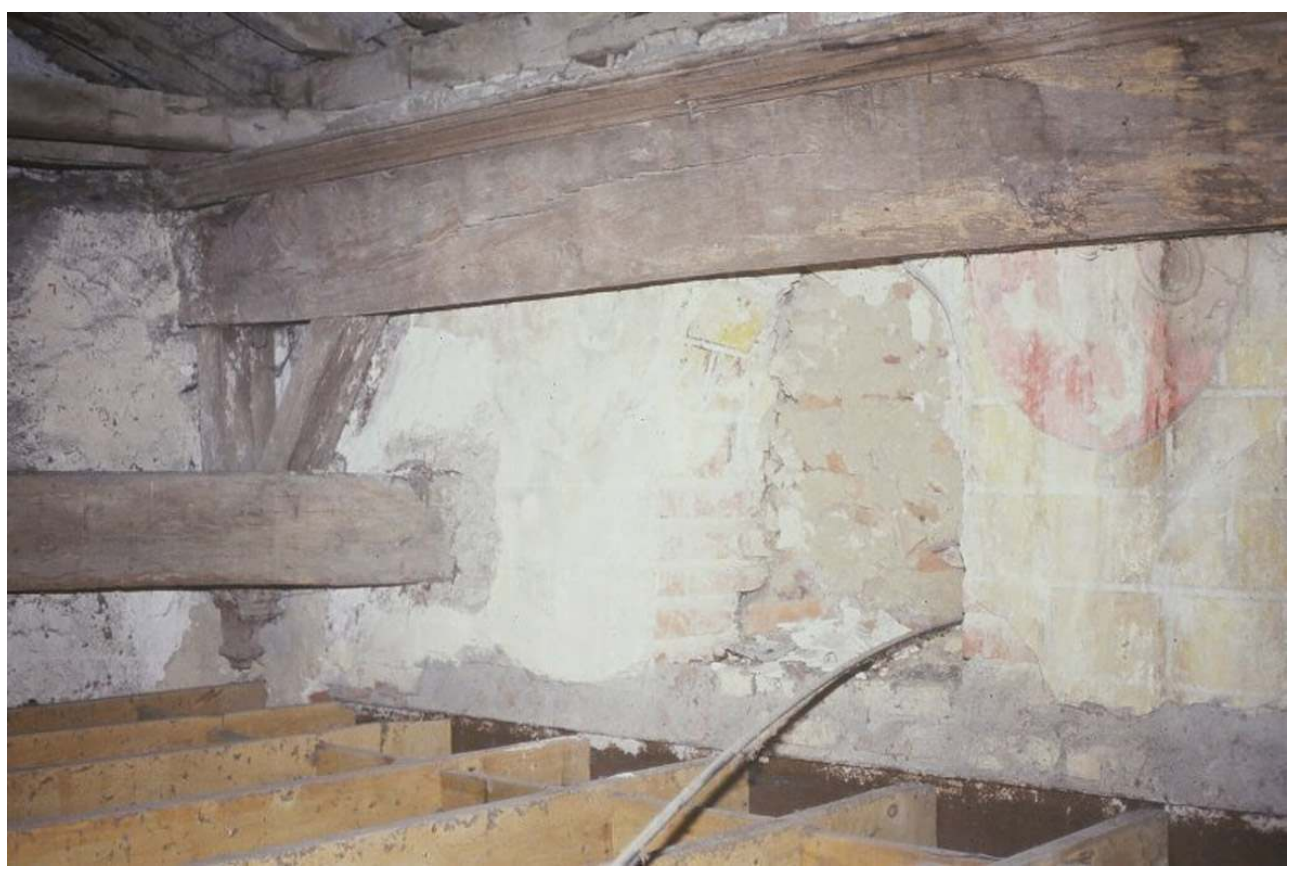

Baie haute occultée, élévation Sud, sous comble actuel

Phot. D. Watin-Grandchamp ( C CRMH Midi-Pyrénées, 1995

22 Ces blasons sont traités comme des écus pendus aux murs par une patte d'attache figurée dans des tons brun-rouges. Ils portent en pointe un petit té bouleté servant aux hérauts d'armes à poser au sol les écus d'apparat. Emaux et meubles sont en cours d'identification, la plupart des armes n'étant connues que par des sceaux non polychromes. On peut dater ces peintures de la fin du XIII ${ }^{e}$ ou du début du XIVe siècle. 
Figure 14

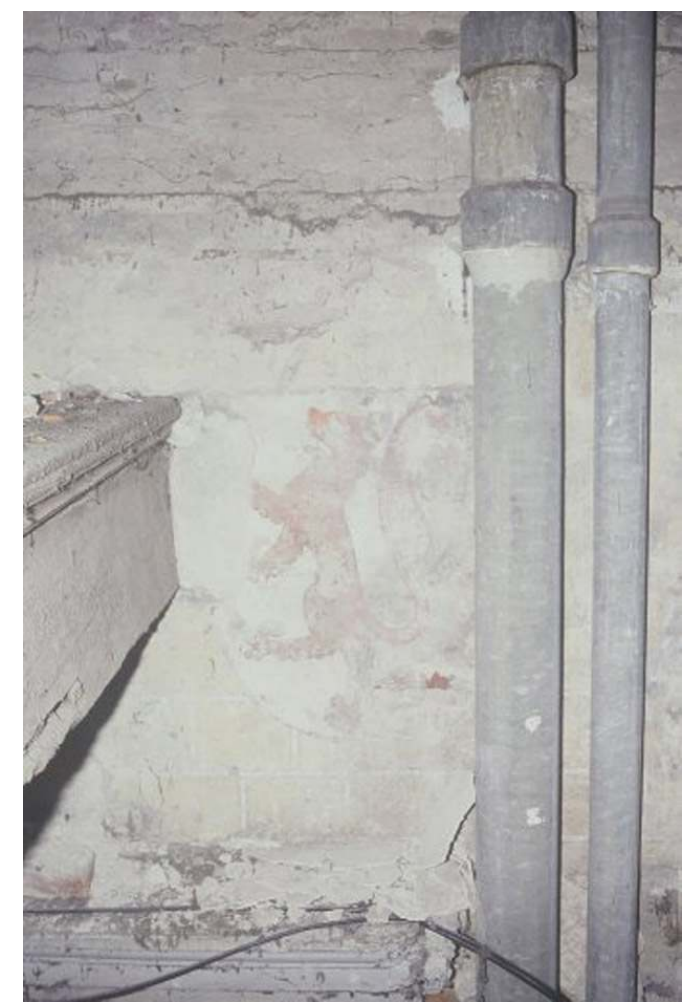

Frise de blasons sous comble : lion hissant à queue biphide des Lautrec

Phot. D. Watin-Grandchamp (c) CRMH Midi-Pyrénées, 1995

On a pu identifier les lions hissant à queue bifide des Lautrec (fig. $\mathbf{n}^{\circ} \mathbf{1 4}^{4}$ ) et les croix vuidées, cléchées et pommelées de la maison de Toulouse avec des inversions d'émaux, ce qui complique l'identification, qui sont le reflet des ramifications et alliances multiples des Lautrec. Les chevrons des Lévis sont présents, à la fin du XIII ${ }^{\mathrm{e}}$ siècle : Béatrix II vicomtesse de Lautrec avait épousé en première noce Philippe I, seigneur de Mirepoix. On $\mathrm{y}$ voit aussi les bandes des comtes de Foix, les lions à château donjonné des Castelnau de Bonafous, autres proches des Lautrec.

24 La partie haute du mur pignon ouest présente des vestiges d'une bande à décor géométrique ondé rouge, blanc et jaune. Au-dessus, l'enduit disparait sur une large bande qui semble correspondre à l'emplacement d'une poutre disparue du couvrement médiéval de la salle. Le sommet du pignon est orné d'un motif à damier ocre-rouge et jaune.

La décoration armoriée de cette "salle haute" est proche de celles que l'on trouve à la même époque à la Tour des Loives à Montfalcon (Isère), au donjon de Touffou à Bonnes (Vienne) ou au château de Ravel (Puy-de-Dôme) entre autres. Ici, elle affirme manifestement le pouvoir des abbesses, toutes issues de la famille de Lautrec.

Elle a été modifiée au $\mathrm{XV}^{\mathrm{e}}$ ou au $\mathrm{XVI}^{\mathrm{e}}$ siècle comme le prouvent les éléments conservés d'un plafond mouluré disposés perpendiculairement au pignon recoupant certains blasons. Cette transformation correspond au moment où la famille Lautrec perd de son influence en Languedoc.

Le dossier de protection au titre des Monuments historiques d'une abbaye des XVII ${ }^{\mathrm{e}}$-XVIII

e siècle a été le révélateur des vestiges médiévaux dissimulés par les élévations de 
l'époque classique. Aujourd'hui, la commune et le conseil général du Tarn sont sensibilisés à la mise en valeur du lieu et le déplacement prévu du collège devrait en faciliter l'étude. Mais le "module médiéval" pose plus de questions qu'il n'apporte de réponses sur l'histoire de l'abbaye médiévale de Vielmur. Seule une étude archéologique approfondie- suite des fouilles du cloître du Moyen Âge dans la cour nord, qui a fait apparaitre des éléments sculptés proches de Moissac et de Saint-Sernin, sondages dans les parties hautes de l'église où l'on a découvert une peinture murale représentant une religieuse, investigations minutieuses dans les divers niveaux du "module médiéval" permettraient sans doute de faire d'autres découvertes. La datation et l'identification de la fonction exacte des dispositions médiévales, conservées lors des travaux des XVII ${ }^{e}$ et $\mathrm{XVIII}{ }^{\mathrm{e}}$ siècles, en dépendent. Alors une véritable monographie sur l'abbaye sera-t-elle possible.

\section{INDEX}

Mots-clés : inventaire général, en ligne, journal, revue électronique, revue numérique, périodique, patrimoine, histoire de l'art, France, Midi-Pyrénées, architecture religieuse Keywords : on line, electronic journal, ejournal, heritage, history of art, France, Midi-Pyrénées, architecture, religious communitie

\section{AUTEUR}

\section{DOMINIQUE WATIN-GRANDCHAMP}

Chargée d'études documentaires, DRAC Midi-Pyrénées Conservation régionale des monuments historiques 1, place Alfonse-Jourdain BP 81131080 Toulouse Cédex 6.

dominique.watin@culture.gouv.fr 